Egyptian

Orthodontic Journal

\title{
EVALUATION OF THE COLOR STABILITY OF DIFFERENT TYPES OF POLYCARBONATE BRACKETS
}

\author{
Nagwa M. Enany ${ }^{1}$; Hanan A. Ismail'; Maha A. Ragab ${ }^{2}$ \\ ABSTRACT: \\ Objective: To investigate the discoloring effect of certain \\ foods and photoaging on the color stability of the three different \\ types of polycarbonate brackets. Materials and Methods: Eighty four \\ brackets were selected and divided into three groups: (1) Composite \\ brackets with ceramic like filler (Silicon brackets); (2) Composite \\ unfilled brackets (Spirit brackets) and (3) Polyoxymethylene brackets \\ (Brilliant brackets). Seven specimens of three different esthetic \\ brackets were exposed to tea, coke, curry and accelerated \\ photogeing device to a xenon lamp to simulate natural day light. \\ The brackets were analyzed at the beginning of the experiment \\ and after 24 and 72 hours and 25 days of exposure. Color \\ measurements were performed by means of Vita Easy shade \\ Spectrophotometer according to CIELCh coordinates lightness, \\ chroma and hue and color changes (ปE) were computed. Results: \\ Almost all investigated brackets showed clinically unacceptable \\ discoloration during in vitro exposure to food dyes and $U V$ light \\ with the exception of the polyoxymethylene brackets which \\ showed nonperceptible color change after storage 25 days in coke \\ and VV. Tea and curry discolor all brackets and showed a sharp \\ increase in discoloration after 24 hour. Most of the brackets \\ became yellower after UV light exposure. Conclusions: These \\ current in vitro findings indicate that even newly developed \\ plastic brackets, consisting of composite materials or modern \\ polymers (polyoxymethylene) may have clinically unacceptable \\ color stability in the long-term.
}

1 - Professor of orthodontics, orthodontic department Alexandria University.

2 - Post graduate student., orthodontic department Alexandria University. 
Egyptian

Orthodontic Journal

\section{INTRODUCTION}

The request for esthetic fixed orthodontic appliances has been increased. More young patients want an esthetic appearance during treatment with fixed appliances. Esthetic bracket materials are plastic or ceramics. ${ }^{1}$ Early attempts to make the appliance more esthetic in appearance resulted in the introduction of plastic brackets which was first reported by Newman in $1969 .^{2}$

Proffit ${ }^{3}$ and Winchester ${ }^{4}$ reported that, although plastic brackets were introduced with considerable enthusiasm, they suffer from that they are not durable enough to hold up in the mouth in the long term because of staining, discoloration, absorption of water and saliva, wear of the brackets and tie-wing fracture.

Swartz found that some problems encountered in plastic brackets were eliminated through recent advances by development of ceramic brackets which were first introduced in 1987 as a more esthetic alternative to their plastic predecessors. ${ }^{5}$ Gottleieb et al noticed that, between 1986 and 1990, the use of ceramic brackets increased from $6.6 \%$ to $88.2 \%$, whereas the use of polycarbonate brackets decreased from $57.8 \%$ to $24.3 \%{ }^{6}$

Arici and Regan reported that, the advantages of ceramic brackets include color stability and great strength. Nevertheless, the use of ceramic brackets may result in problems with excessive bond strength, damage to the enamel during removal, and bracket breakage because of brittleness. ${ }^{7}$ Therefore, Brantley and Eliades found that current research on reinforcement methods of plastic brackets, including reinforcement of the polymer by fillers (so- called'composites') or fibers, or the use of metallic inserts on the bracket slot. ${ }^{8}$

The color stability of plastic brackets was found to depend on various factors, such as filler level and type of discoloration. ${ }^{9}$ Color stability of brackets is due to exogenous and endogenous factors. The reason for endogenous discoloration can be found in ultraviolet (UV) irradiation and thermal energy. UV light can induce physico-chemical reactions in the polymer, causing irreversible color changes of the brackets. Exogenous influences are foods that stain and even colored mouth rinses . $^{10-13}$

Volume 41 - June 2012 
In the present study, the influence of the long term photoageing and food colorants on the color stability of the three different types of plastic brackets was tested. Hypothetically, the ceramic filler should reduce staining of the brackets and also reinforce the plastic bracket. On the other hand, the metal slot should prevent distortion during torque force application and reduce bracket friction with the metal arch wire. ${ }^{4}$

\section{MATERIAL AND METHODS}

Eighty four plastic orthodontic brackets from three different companies were exposed to tea, coke, curry and UV light for 24 hours, 72 hours and 25 days at $37^{\circ} \mathrm{C}$. The color stability of each bracket was evaluated by the color difference before and after its immersion in different color affecting media with a Vita Easyshade Spectrophotometer device according to CIEL Ch (Commission Internationale de l'Eclairage) coordinates lightness, chroma and hue.

The brackets were divided into three groups ( 28 per bracket group):

1. Group I: Silicon Brackets*. They were composed of plastic with ceramic like filler. (Figure 1)

2. Group II: Brillant Brackets ${ }^{* *}$. They were composed of polyoxymethylene material. (Figure 2)

3. Group III: Spirit MB Brackets ${ }^{* * *}$. They were composed of composite with stainless steel reinforced slot. (Figure 3)

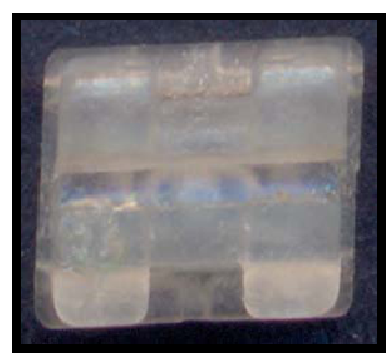

Figure 1

Silicon Bracket

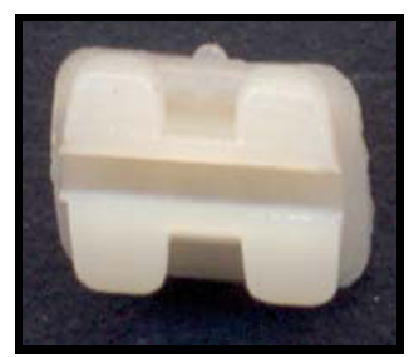

Figure 2

Brillant Bracket

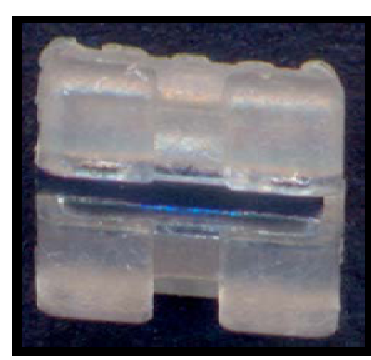

Figure 3

Spirit Bracket

\footnotetext{
* American Orthodontic, USA.

** Forestadent, Germany.

*** Sybron Dental Specialties Ormco, USA.
} 
Methods: A black $3.5 \mathrm{~cm}$ circular sample holder with $4 \mathrm{~mm}$ diameter window in the center for each type of the brackets was fabricated with self curing acrylic resin to position the brackets in standardized settings.

All brackets were rinsed with running water; air dried and then color measurements taken as a baseline measurements using the self-calibrating electronic tooth-color measurement device VITA Easyshade (Figure 4).

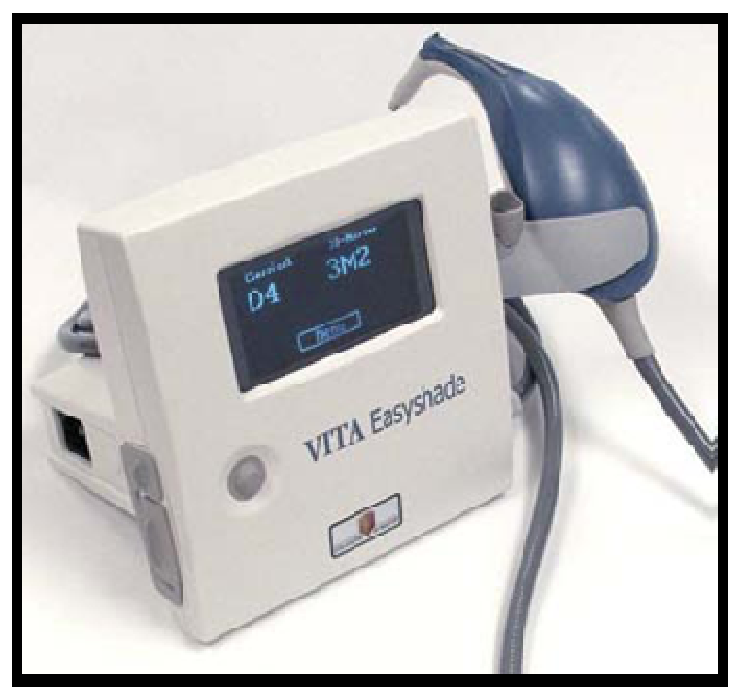

Figure 4: Vita Easy Shade Spectrophotometer

Seven brackets per group were placed in a glass container with one of the three media at $37^{\circ} \mathrm{C}$ and allowed to soak for 24 hours. Another seven brackets of each group underwent accelerated photoageing in an irradiation chamber to investigate the influence of UV irradiation on the brackets for 24 hours. They were then removed, rinsed; air dried and color readings taken. After the 24 hours, the brackets soaked again in the solutions and exposed to UV irradiation for 72 hours and then removed, rinsed, air dried and color measurements taken. After that, the brackets exposed to all media for 25 days and then color measurements were taken in the same way. 
All measurements were done in the internationally standardized CIELAB color space. ${ }^{14}$ The changes in the lightness and chromaticity coordinates

$\left(\Delta \mathrm{L}^{*}, \Delta \mathrm{a}^{*}\right.$ and $\left.\Delta \mathrm{b}^{*}\right)$ as a result of exposure to diet substances were determined first and the color difference $(\Delta \mathrm{E})$ was calculated using the relationship:

$$
\Delta E=\left[\left(\Delta L^{*}\right) 2_{+}\left(\Delta a^{*}\right) 2_{+}\left(\Delta b^{*}\right) 2\right] 1 / 2
$$

Ruyter et al ${ }^{15}$ reported that a shift in $\Delta \mathrm{E}$ value of $>3.3$ reflects a change that is a clinically significant visual discoloration.

\section{Statistical Data Analysis:}

The statistical analysis was done using Statistical Package for Social Sciences (SPSS, version 15.0). Descriptive statistics were displayed for values of color change $(\Delta \mathrm{E})$ between different groups at different follow up periods as mean, median and standard deviations. Normality testing was done using Kolmogrov Smirnov test and values were found to be normally distributed. Chi square test was used to compare values between different groups followed by Fisher's Exact Test probability for statistically significant differences. Significance level was set at 5\%. Simple linear Regression Equation was used to compare trends of delta $\mathrm{E}$ for the different brackets in different media. Boxplot chart was used for graphical presentation.

\section{RESULTS}

There was statistically significant color change between the brackets after overall comparison to all media at 25 days. (Table I, Figure 5) The Brillant brackets had the least median value of $\Delta \mathrm{E}$ in comparison with the other bracket groups $(\Delta \mathrm{E}=8.5)$. The Silicon brackets showed greater color change followed by Spirit brackets which were $(\Delta \mathrm{E}=11.7),(\Delta \mathrm{E}=11.6)$ respectively. 
Egyptian

Orthodontic Journal

Table I. Overall comparison of $\Delta \mathrm{E}$ between bracket groups at 25 days

\begin{tabular}{|l|l|l|l|l|}
\hline \multicolumn{1}{|c|}{ Brackets } & \multicolumn{1}{|c|}{ Median } & \multicolumn{1}{|c|}{ Mean } & Std. Deviation & Median Test \\
\cline { 1 - 4 } 1 Silicon & 11.7 & 14.7 & 8.2 & \\
\cline { 1 - 3 } 2 Brillant & 8.4 & 14.6 & 15.5 & \multirow{2}{*}{$\square^{2}=1.143$} \\
\cline { 1 - 3 } 3 Spirit & 11.6 & 12.6 & 5.6 & $\mathrm{P}=0.565$ \\
\hline
\end{tabular}

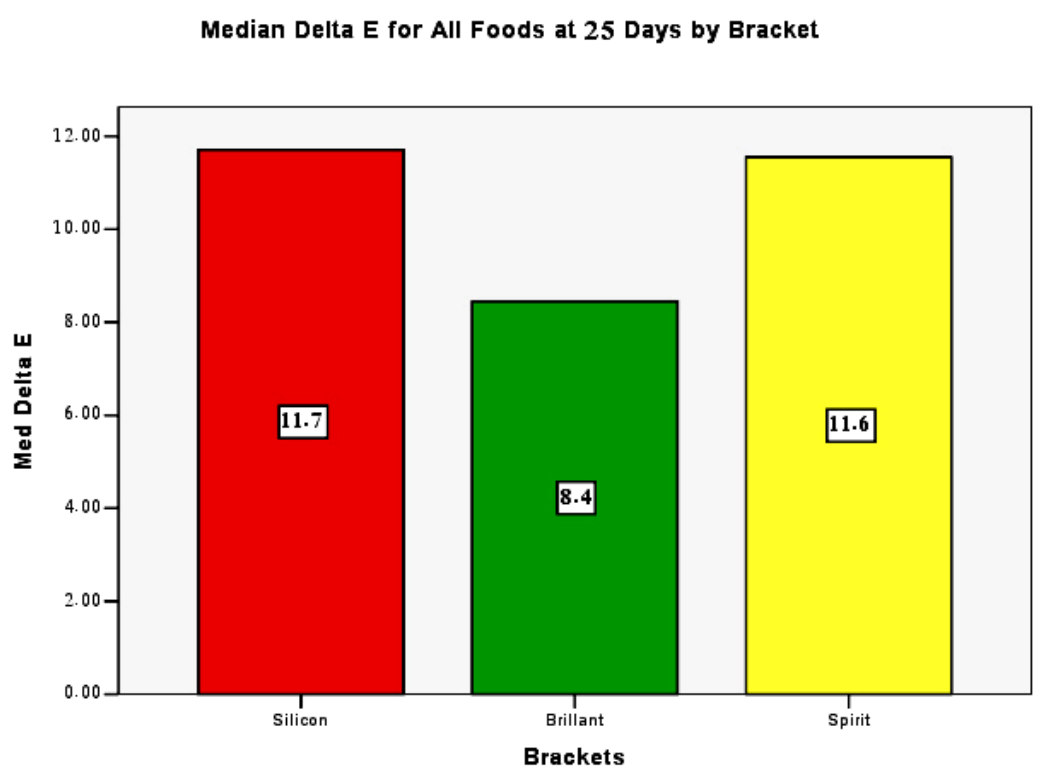

Figure 5. Overall comparison of $\Delta \mathrm{E}$ between bracket groups at 25 days for all foods.

In this study, we were interested in observing whether the tested brackets showed perceptible color changes rather than just statistically significant color differences. Color changes that are perceptible may compromise the clinical acceptability of the brackets. Ruyter et al ${ }^{15}$ considered that the values of $\Delta \mathrm{E}$ greater than or equal to 3.3 are visually perceptible and clinically unacceptable. As testing began, a light 


\section{Egyptian}

Orthodontic Journal

discoloration on the brackets was observed; later, by enhancing the exposure time from 24 to 72 hours, all brackets showed a significant increase of $\Delta \mathrm{E}$ values and a discoloration on the brackets was observed. At the end of testing after 25 days, all bracket groups achieved a certain saturation of discoloring, the brackets became darker and they also represented clinically significant changes as was evident from Ruyter criteria. (Figures 6-9)

A
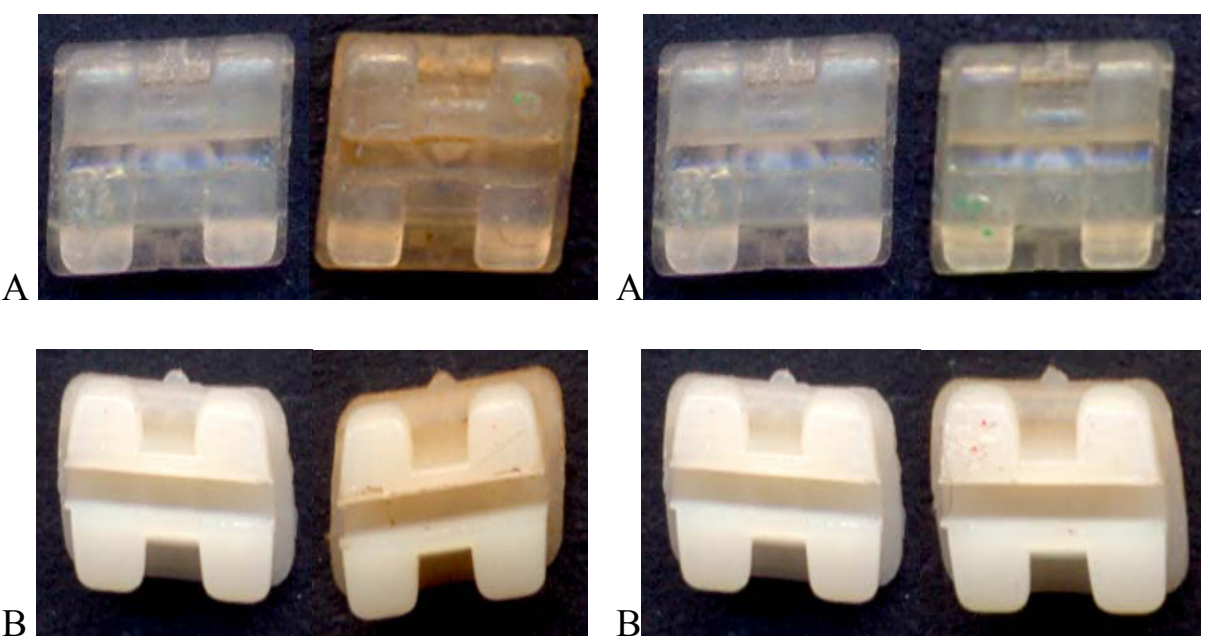

$\mathrm{C}$
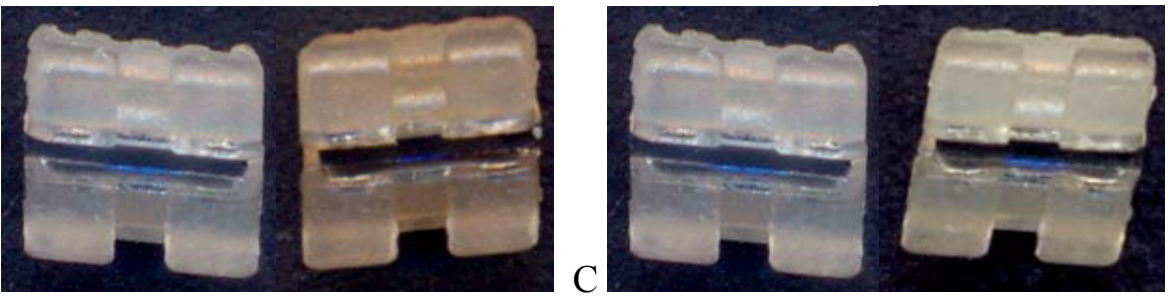

Figures 6. Comparison of control brackets (on the left) to those immersed in tea

Figures 7. Comparison of control brackets (on the left) to those immersed in coke (on the right) on day 25: a) Silicon brackets, (on the right) on day 25: a) Silicon brackets, b) Brillant brackets, c) Spirit brackets

b) Brillant brackets, c) Spirit brackets.

Volume 41 - June 2012 


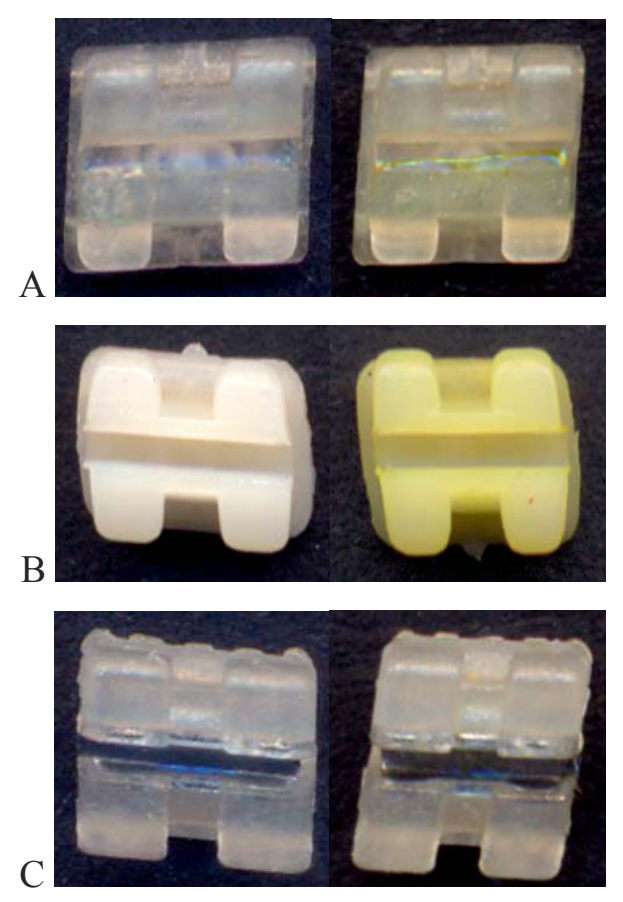

Figures 8. Comparison of control brackets (on the left) to those immersed in curry (on the right) on day 25: a) Silicon brackets, b) Brillant brackets, c) Spirit brackets.
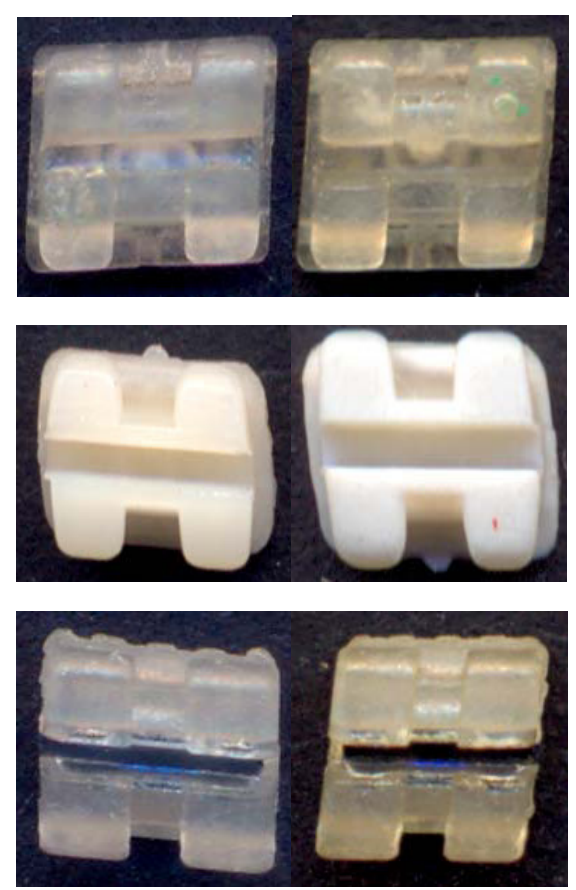

Figures 9. Comparison of control brackets (on the left) to those immersed in UV (on the right) on day 25: a) Silicon brackets, b) Brillant brackets, c) Spirit brackets.

\section{DISCUSSION}

The present study was designed to evaluate the color stability of esthetic plastic brackets during exposure to food dyes and UV irradiation over time intervals of 24,72 hours and 25 days and determine clinically perceptible color changes $(\Delta \mathrm{E})$ between different plastic brackets.

Color stability is an important parameter for modern esthetic brackets. Color changes can be distinguished by a colorimeter, spectrophotometer or visually.

It was necessary to create standard measurement criteria for three-dimensional brackets. This is why the Easyshade measuring probe was chosen because the probe thoroughly shields the area to be measured from external light. ${ }^{16}$ The advantages of CIELAB system for color 
Egyptian

Orthodontic Journal

measurement is that it more closely represents human sensitivity to color and equal distances in this system approximately equal perceived color differences. ${ }^{17}$

Numerous tests have been used for artificial aging of bracket materials to investigate color stability in vivo and in vitro. As clinical testing is difficult to carry out and results may not be comparable due to a combination of miscellaneous factors, an in vitro investigation was undertaken. $^{18}$ Various techniques have been described to study discoloration of orthodontic materials. The solutions used in this study which were coke, curry, tea and accelerated photoageing, do not consider all substances to which bracket materials may be exposed, though they have been used in similar research ${ }^{16}$ and represent media common to the oral environment. It has been shown that the type of immersion solution can affect the degree of color change, with curry and tea often contributing to the most significant staining. ${ }^{19}$

Several studies concluded that a period of 24 hour artificial treatment is too short to investigate discoloration of dental materials. ${ }^{20}$ Since this work complements previous studies, that extension of the exposure time to 25 days was considered suitable to examine the color stability of the plastic brackets.

Coupled with the previous finding that measured color change $\Delta \mathrm{E}$ increased from 24 hour to 72 hours and 25 days, it can be concluded that brackets discoloration progresses with immersion time increase rather than bracket materials from which they are made.

In this study, we were interested in observing whether the tested brackets showed perceptible color changes rather than measuring the statistical significance of color differences. This depends in part on the patient's idea of what is esthetically attractive and also on the patient's perceptive abilities. According to Ruyter et al, ${ }^{15}$ a $\Delta \mathrm{E}$ of 3.3 is visually perceptible and therefore clinically unacceptable, all tested brackets had perceptible color changes after exposure to all media except Brilliant brackets which showed nonperceptible color change after storage 25 days in coke and UV.

Volume 41 - June 2012 
Egyptian

Orthodontic Journal

Satisfactory color stability was measured for the Brillant brackets after coke and UV irradiation. The Brillant brackets consisting of polyoxymethylene53, was the only bracket that showed a highly visible discoloration towards the bright yellow color of curry after $24 \mathrm{~h}$.This can be explained by the fact that the Brillant bracket already has a more yellowish color due to manufacturing conditions. This was in agreement with the study done by Susanne Wriedt et al ${ }^{16}$ in which they showed that Brillant bracket, unlike all the other materials, becomes deeply discolored in curry and they thus recommend that, for esthetic reasons, such brackets are unsuitable for patients who enjoy curry.

The Spirit brackets consisting of composite showed undesirable discoloration after 24hours from storage in all media. According to Janda et $\mathrm{al}^{21}$, the resin matrix itself influences color stability. Rosentritt et al reported no synergetic effects on color behavior after UV ageing and storage in food dyes. ${ }^{22}$ Silicon brackets consisting of composite with ceramic like filler seem to be less susceptible for endogenous discoloration than the Spirit bracket. This might explain why highly filled composite brackets had less endogenous discoloration than unfilled brackets. $^{23}$

Color stability is only a variable that must be considered when choosing a bracket material, but it may be of great importance to patients and clinicians when working in the esthetic zone. In some patients, the color change is very minute and not perceptible by the patient while in other patients, it is huge and noticed by the patient depending on the patient's diet and hygiene habits. Clinical trials and experience are required to assess whether particular bracket materials will meet the esthetic demands for color stability in a clinical setting. Despite our results, the clinical performance of the bracket depends on various synergistic effects in the oral environment that cannot be simulated precisely by in-vitro investigations.

\section{REFERENCES}

1. Julia K, Andreas F, Michael B, Peter P. Evaluation of alternative polymer bracket materials. Am J Orthod Dentofacial Orthop 2010; 137:362-7. 
2. Newman GV. Adhesion and orthodontic plastic attachments. Am J Orthod 1969; 56:573- 88 .

3. Proffit WR, Fields HW.Contemporary Orthodontics. Louis. Mosby, 2nded; 1986.

4. Winchester LJ. Aesthetics brackets: To perfect or to reject? Dent Update 1992; 19:107-14.

5. Swartz M L .Ceramic brackets . Journal of Clinical Orthodontics 1988; 22: 82-88.

6. Gottlieb EL, Vogels DS. Study of orthodontic diagnosis and treatment procedures part I resulted trends. J Clinical Orthod 1991; 25:145-156.

7. Arici S, Regan D. Alternatives to ceramic brackets: the tensile bond strengths of two aesthetic brackets compared ex vivo with stainless steel foil-mesh bracket bases. British Journal of Orthodontics 1997; 24: 133-137.

8. Brantley WA, Eliades T. Orthodontic materials: scientific and clinical aspects. Thieme, Stuttgart, Germany ;2001.

9. Andreas F, Michael B, Dieter M. Esthetic brackets: The influence of filler level on color stability. Am J Orthod Dentofacial Orthop 2007;132:5.e13-5.e16.

10. Khokhar ZA, Razzog ME, Yaman P. Color stability of restorative resins. Quintessence Int 1991; 22:7-733.

11. Seher J, Viohl J. In vitro discoloration of experimental and dental resins due to the effect of dyes and UV irradiation. Dtsch Zahnarztl Z $1992 ; 47: 6-634$.

12. Dietschi D, Campanile G, Holz J, Meyer JM. Comparison of the color stability of ten new generation composites: an in vitro study. Dent Mater 1994; 10:62-353.

13. Leibrock A, Rosentritt M, Lang R, Behr M, Handel G. Color stability of visible light curing hybrid composites. Eur J Prosthodont Restor Dent 1997;5:30-125. 
14. Commission Internationale de l'Eclairage(CIE). Light as a true visual quantity: principles of measurements.CIE Pub, Vienna, Austria, Bureau, central de la CIE 1978;41.

15. Ruyter IE, Nilner K, Moller B. Color stability of dental composite resins for crown and bridge veneers. Dent Mater 1987; 3: 246-51.

16. Wriedt S, Schepke U, Wehrbein H. The discoloring effects of food on the color stability of esthetic brackets. J Orofac Orthop 2007;68:308-320.

17. Billmeyer FW, Saltzman M. Principle of color technology. 2nded. New York: John Wiley \& Sons 1981;19:1-110.

18. Arthur SK, Frederick CS, John C, Tak WC. Color stability of provisional prosthodontic materials. Journal of Prosthetic Dentistry 2004 ;91: 447- 452.

19. Scotti R, Mascellani SC, Forniti F. The in vitro color stability of acrylic resins for provisional restoration. Int $\mathrm{J}$ Prosthodont 1997;10:164-168.

20. Andreas F, Michael B, Dieter M. In vitro color stability of aesthetic brackets. European Journal of Orthodontics 2007;29: 354-358.

21. Janda R, Roulet JF, Latta M, Steffin G, Ruttermann S. Color stability of resin-based filling materials after aging when cured with plasma or halogen light. Eur J Oral Sci 2005;113: 251-7.

22. Rosentritt M, Lang R, Behr M, Handel G. In vitro discoloration of restorative materials caused by UV light and food colorants. Acta Odontol Scand 1999;4:147-52.

23. Eldiwany M, Friedl KH, Powers JM. Color stability of lightcured and post-cured composites. Am J Dent 1995;8:179-181. 